\title{
An Overview of the Management of Flexor Tendon Injuries
}

\author{
M. Griffin ${ }^{1}$, S. Hindocha ${ }^{*}, 2,3$, D. Jordan ${ }^{3}$, M. Saleh ${ }^{4}$ and W. Khan ${ }^{5}$ \\ ${ }^{I}$ Academic Foundation Trainee, Kingston Upon Thames, London, UK \\ ${ }^{2}$ Department of Plastic Surgery, Whiston Hospital, Warrington Road, L355DR, UK \\ ${ }^{3}$ Department of Plastic Surgery, Countess of Chester Hospital, Liverpool Road, Chester, CH21UL, UK \\ ${ }^{4}$ Ain Shams University, Khalifa El-Maamon St, Abbasiya Sq, Cairo 11566, Egypt \\ ${ }^{5}$ University College London Institute of Orthopaedics and Musculoskeletal Sciences, Royal National Orthopaedic \\ Hospital, Stanmore, Middlesex, HA74LP, UK
}

\begin{abstract}
Flexor tendon injuries still remain a challenging condition to manage to ensure optimal outcome for the patient. Since the first flexor tendon repair was described by Kirchmayr in 1917, several approaches to flexor tendon injury have enabled successful repairs rates of $70-90 \%$. Primary surgical repair results in better functional outcome compared to secondary repair or tendon graft surgery. Flexor tendon injury repair has been extensively researched and the literature demonstrates successful repair requires minimal gapping at the repair site or interference with tendon vascularity, secure suture knots, smooth junction of tendon end and having sufficient strength for healing. However, the exact surgical approach to achieve success being currently used among surgeons is still controversial. Therefore, this review aims to discuss the results of studies demonstrating the current knowledge regarding the optimal approach for flexor tendon repair. Post-operative rehabilitation for flexor tendon surgery is another area, which has caused extensive debate in hand surgery. The trend to more active mobilisation protocols seems to be favoured but further study in this area is needed to find the protocol, which achieves function and gliding but avoids rupture of the tendons. Lastly despite success following surgery complications commonly still occur post surgery, including adhesion formation, tendon rupture and stiffness of the joints. Therefore, this review aims to discuss the appropriate management of these difficulties post surgery. New techniques in management of flexor tendon will also be discussed including external laser devices, addition of growth factors and cytokines.
\end{abstract}

Keywords: Flexor tendon repair, flexor laceration, post operative mobilisation, hand injuries, tendon injuries.

\section{INTRODUCTION}

Tendons connect muscle to bone to allow the force to be transmitted from the muscle to the bone, allowing the joint to move. Tendons are composed of approximately $90 \%$ to $95 \%$ of tenoblasts and tenocytes [1], with the remaining 5-10\% consisting of chrondrocytes at the bone attachment and insertion sites, vascular cells, capillary endothelial cells and smooth muscle cells of the arterioles. Tenoblasts are immature tendon cells, which mature into tenocytes. Collagen type 1 account for $65-80 \%$ [2-4] of the dry mass of tendons. Collagen starts as triple-helix polypeptide chains, which unties into fibrils, then fibres, fascicles, tertiary bundles which lastly forms the tendon [5-7] (see Fig. 1). The epitenon is a connective-tissue sheath containing the vascular, lymphatic and nerve supply to the tendon and covers the whole tendon 6 . The epitendon goes deep within the tendon bundle to covers the tertiary bundles to form the endotendon [6]. The endotendon is connective tissue covering each tendon fibre and surround by paratendon which is connective tissue containing type I and type III

*Address correspondence to this author at the Department of Plastic Surgery, Whiston Hospital, Warrington Road, L355DR, UK;

Tel: 01244366265; Fax: 01244366265; hindocha2001@yahoo.com collagen fibrils, elastics fibrils and the synovial cells [8]. Synovial sheaths are formed where there is increased stress and consist of an outer fibrotic sheath and inner synovial sheath, which contains visceral and parietal sheets [9]. The inner synovial sheath produces synovial fluid. The synovial sheaths condense at certain places along the synovial sheaths of the fingers to create an arrangement of five annular pulleys and three cruciform pulleys to acts as fulcrums for the tendons. There are two flexor tendons to each finger and one for the thumb (see Fig. 2). The hand is divided into 5 zones Zone I starts at the end of the fingers with zone $\mathrm{V}$ at the wrist joint. Zone 1 is distal to the flexor digitorium superficilias insertion. Zone II (no mans land) between the limits of the flexor tendon sheath which is at the A1 pulley and the insertion of the flexor digitorum superficialis, which is in close proximity to the flexor tendon sheath. Zone III is from the distal edge of the transverse carpal ligament and the A1 pulley, with nerves, tendons and vessels being frequently involved due to the close proximity. Zone IV refers to the tendons within the carpal tunnel and zone $\mathrm{V}$ encompasses the forearm proximal to the carpal tunnel [10] (see Fig. 2). At the myotendinous junction the tendinous collagen fibrils insert into the myocyte processes, which transmits the forces by the muscles fibres to be conveyed to the collagen fibrils $[11,12]$. The osteotendious junction consists of tendon, 
firbocartilage, mineralized firbocatrilage and bone, which prevents collagen failure and shearing [13].

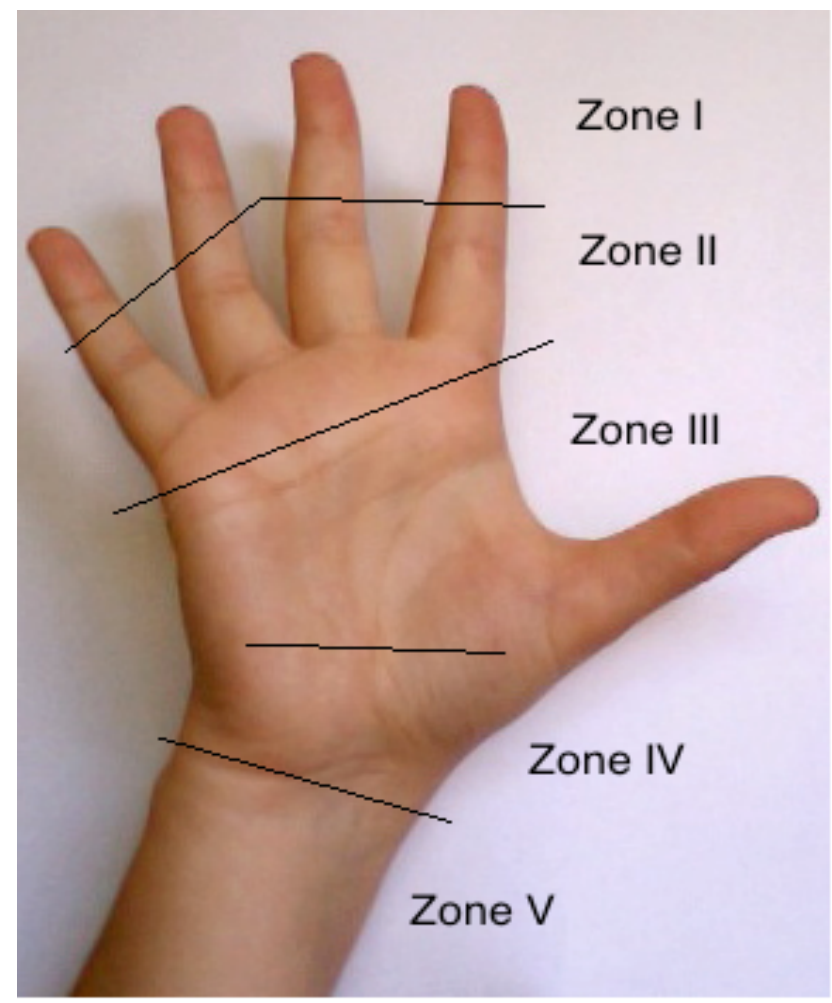

Fig. (1). Anatomy of tendons.

Tendons are perfused by the longitudinal vessels that enter in the palm and extend down the intratendinous channels, from vessels that enter at the level of the proximal synovial fold, the segmental branches or from the paired arteries that enter in the tendon sheaths by means of the long and short vincula and vessels that enter directly at the flexor digitorum superficialis and the flexor digitorum profundus at the osseous insertions. Synovial fluid is also nutritional as well as lubricating mechanism for tendons. Fluid is forced into the interstices of the tendon though small conduits in the tendon surface as the digit is flexed and extended.

The way in which tendon heals has been researched over the years. In summary tendon healing involves an inflammatory stage in 48 to 72 hours where erthyrocytes and inflammatory cells including neutrophils enter the site of injury to initiate angiogenesis increase vascular permeability and stimulate tenocyte proliferation and recruitment of other inflammatory cells. Then a proliferation stage occurs where repairing fibroblastic and collagen-producing cells enter from 5 days to 4 weeks. Lastly the remodelling takes place for approximately after 6 weeks where the tissue repair changes from cellular to fibrous [14]. The tenocyte metabolism remains high and the tenocytes and collagen fibres become aligned [15]. Later in this stage there is a gradual change of the fibrous tissue to the scar like tendon tissue over approximately one year [15]. Flexor tendons heal by both intrinsic and extrinsic mechanisms. The intrinsic method includes the nutrients that are supplied by the synovial fluids and the proliferation of the epitenon and endotenon tenocytes. The extrinsic part is the fibroblastic response of the sheath and the tissue and the cells surrounding the tendon. The relative contribution from each part depends on the region of origin of the tendon, the trauma and the amount of stress induced by motion after repair as occurred. The weakest point of tendon healing is 5 to 10 days postoperative, which should be thought out in postoperative management plans.

\section{FLEXOR TENDON INJURY}

Flexor tendon injuries are a common event as the tendons lie close to the skin and so are usually the result of either lacerations such as those from knives or glass, from crush injuries and occasionally they can rupture from where they

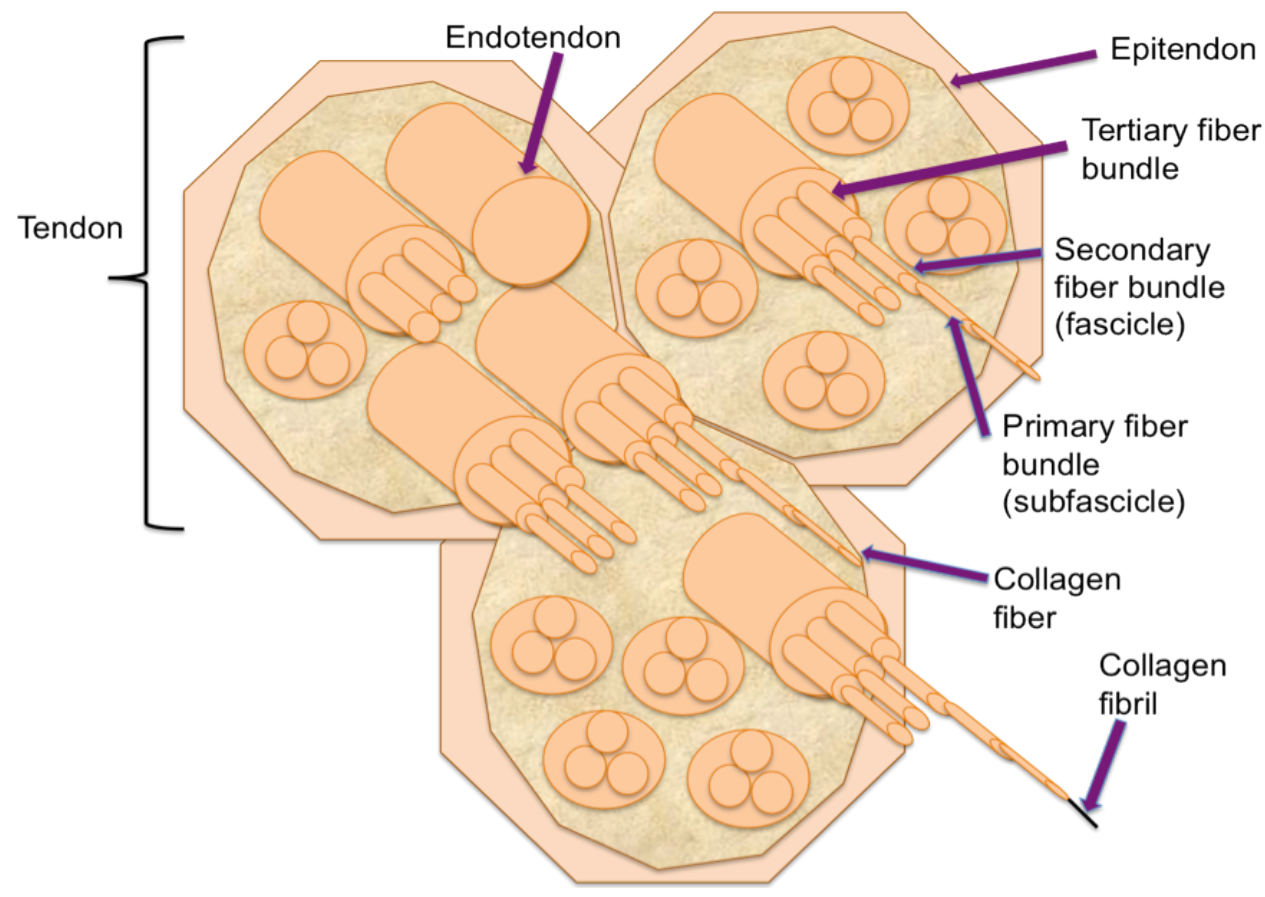

Fig. (2). Flexor Zones of the hand. 
are joined at the bone during contact sports such as football, rugby and wrestling. Flexor tendon injuries are a challenging problem for orthopaedic surgeons due to three main reasons. Firstly, flexor tendon injuries of the hands are a clinical problem because they cannot heal without surgical treatment, as the two ends need to be surgically brought together for the healing to occur unlike other tendons including the Achilles tendon where it could be placed into plantar flexion to heal. Secondly postoperative management needs to be carefully planned as mobilisation has shown to be essential to prevent adhesions and improve gliding but this can risk rupture. Lastly due to the unique anatomy of the tendons running through flexor tendon sheaths to function, surgeons need to plan preventing increasing the bulkiness of the tendon through its sheath, which is not always possible from scarring as this affects the functional outcome of the tendon.

\section{PRESENTATION OF FLEXOR TENDON INJURY AND ASSESSMENT}

The symptoms that a patient will present with if the person has a flexor tendon injury are include not being able to bend the finger, pain when bending the finger or localised swelling and open cuts. Tendon injuries can occur in all 5 zones of the hand.

As with any hand condition history and physical examination needs to uncover both primary and secondary damage. Like in any other hand injury, age gender, mechanism and nature of injury, time elapsed are allimportant factors that will affect the planning of and decisions made at the time of the repair. The mechanism of injury is vitally important to understand as knowing the level of contamination (clean knife versus oily scrap yard machine) will indicate preoperative and postoperative care. Furthermore knowing in what position is the finger was during the hyperextension and extension of the finger during injury can give a clue to the findings at surgery so the surgeons can prepare for surgery with the correct approach. Also looking for previous injuries can be useful to tell the patient the realistic outcomes of surgery.

Physical examination needs to include a full examination of both hands and must be carried out systematically. The flexor digitorum superficialis and the flexor digitorium profundus tendons should be tested individually. A normal intact flexor digitorum superficialis is indicated when all the adjacent digits are held with all joints in extension while the patient flexes the finger at the proximal interphalangeal joint. On the other hand if the middle phalanx is in extension while the patient is directed and able to flex the distal interphalangeal joint, the flexor digitorium profundus tendon has shown to be intact. Fracture should be confirmed using radiographs before surgical intervention, as during surgery these will need to be reduced and fixed before vascular or tendon repair. Loss of underlying skin and soft tissue needs debridement so this needs to be identified as well as the planning of flaps. Nerve damage is vital to assess, whether it is at the level of the arm, wrist or digit. The ulnar, median and radial nerve needs to be tested by the preference of choice chosen by the surgeon. Vascular compromise needs to be recorded by the presence or absence of pulses and capillary refill. The ischaemic digit or limb provides an increased level of urgency.

\section{FLEXOR TENDON REPAIR}

The decision to repair a tendon needs to take into account many variables. Primary surgical repair results in better functional outcome compared with secondary tendon repair (more than 3 weeks after the primary injury) or tendon graft surgery for repairing flexor tendon injuries [16]. After 3 weeks primary tendon repair will not be possible because of proximal tendon end swelling, tendon contraction and muscle fibrosis. Secondary repair is still acceptable for the injured tendons that cannot be repaired primarily [16], which may be due to increased risk of infections or excessive loss of soft tissue. Usually crush or avulsion repair require urgent repair. To date there is no universally accepted gold standard for type of suture material or suture technique used to repair flexor tendon injuries with surgeons using their chosen method to repair such injuries (see Fig. 3). Flexor tendon repair was first described by Kirchmayr in 1917, when he published a method of 'locking' suture for tendon repair [17]. However, since then, several surgical approaches have been described. Most authors show that more than $60 \%$ of the flexor tendon should be cut before surgeons offer surgical intervention.

Good exposure is vital to ensure good repair of the tendons it need to enable the surgeon to retrieve the cut tendons, maintain vascularity and not cause contractures and provide access to asses the wound accurately by starting away from the zone of injury and then proceeding to the injury. The type of incision by the surgeons used does depend on surgeon choice but the most commonly incisions used are the bruner zig-zag and the bunnell incision [18]. The bruner zig-zag incision avoids the digital neurovascular bundles and stays lateral to the flexion creases and the bunnell is a midlateral incision which avoids the volar surface running in parallel to the digital neurovascular bundles [19]. Both are usually used as the bunnell avoids disturbing sensation to the volar aspect and the brunner zigzag avoids vascular compromise.

Strickland stated that the ideal tendon repair should have minimal gapping at the repair site, minimal interference with tendon vascularity, secure suture knots, smooth junction of tendon end and have sufficient strength for healing [20]. Several studies have tried to investigate the most effective surgical approach. The strength of the repair has shown to be affected by the type, number and location of loops the surgeon uses and the type of suture material [21-27].

Bone and joint structures are repaired first, followed by tendons then neurovascular compartments, which are the most delicate structures and therefore left till then end. After the incision has been made, the next step is tendon retraction. Tendon retraction will be prevented if the vincula is intact. If the vincula is not intact then a non crushing clamp should be applied to the lacerated end to facilitate the coming together of the ends [28]. Handling of the tendons should be done with as little trauma as possible to prevent scaring and adhesion. Proximal incision in the flexor sheath can help find the primal tendon when they are not easily visible. Flexing the wrist and 'milking' the forearm in a proximal to distal fashion can enhance the delivery of the retracted tendon. The tendon when identified is then fed through the flexor sheath with a flexible tube or a tendon passer. The proximal stump is then secured with a small gauge needle. 


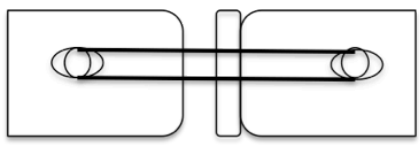

A: Tsuge
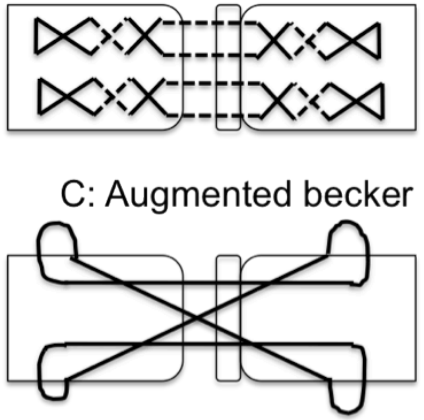

E: 4-strand cruciate

Fig. (3). Common flexor tendon repair techniques.

The tendon repair should be regarded as a companion of core and peripheral sutures, with both contributing to the strength of the repair [29]. Many studies have shown that the strength of a flexor tendon repair proportional to the number of sutures crossing the repair site [30]. Two strand repair techniques were the most commonly used technique in flexor tendon repair up until ten years ago but the discovery that more suture leads to increased strength created a different trend [31, 32]. A number of 4-, 6-, 8 -strand techniques are used in clinical practice [33-35]. The first multi-strand was introduced by savage who used 6-strands across the repair site which showed improved gap resistance and forces and able to withstand early motion [30]. Multi-strand with 4- and 6- core sutures with single-stranded suture have also been investigated which have shown increased gap resistance and fatigue strength compared to 2 strand techniques [36-40]. Eight strand suture has also been compared against 2 six stand savage and 2 strand Kessler and Tajima and was found to have superior strength [41].

A recent study compared a six strand (three figure of eight sutures), four strand (locked cruciate repair), and the combined technique (a 10-strand cruciate repair using both the figure of eight and cruciate sutures) using adult sheep tendons [42]. Biochemically the combine repair was found to be the strongest regarding both gap and failure using single cyclic tensile failure.

Double stranded sutures have also been researched to perform multi strand techniques. Barrie et al., found that increasing the number of stranded from -4 to -8 by using single or double suture in the cruciate non locked and cruciate cross locked configurations and though material strength increased gap resistance did not [43]. Multi strands are more demanding and there are potential risks because they need more tendon handling as you pass the needle through many times which could lead to uneven loading of the tendon.

Loop configuration was first described by Pennigton to describe the precise relation of the longitudinal and transverse strands in the grasping and locking modified Kessler repairs [44]. It has been shown that locking loops improve force and gap resistance compared to grasping loops

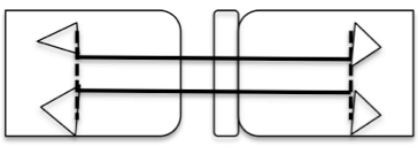

B: Modified Kessler

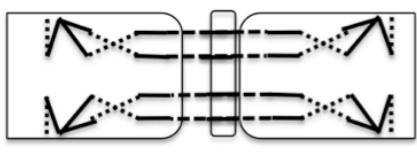

D: 4-strand savage

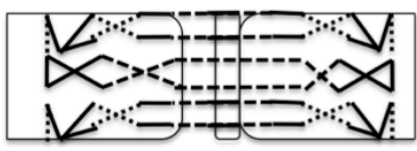

F: 6-strand savage

in flexor tendon repair but the advantage disappear with 3.0 or heavier core sutures as the holding capacity of the suture grips of the tendon healing is then impaired [45]. Furthermore it has also been described that increasing the cross sectional area from 5 to $15 \%$ with the Modified Pennington technique can improve force, but anymore than that and there is no improvement in strength and gap formation increases [46].

The amount of tendon that is involved in the repair is determines by the core suture length. The core suture component of a flexor tendon repair has been shown to provide the first resistance to gap formation and repair failure. With 2- and 4- strand locking and grasping configurations the optimal range of core suture has been shown to be $1.0 \mathrm{~cm}$ with increased gap force, ultimate force and stiffness with this purchase but greater than $1 \mathrm{~cm}$ and 0.4 $\mathrm{cm}$ causes weaker repairs [47, 48]. Repairs with $1.0 \mathrm{~cm}$ core suture purchase failed by suture breakage and $0.4 \mathrm{~cm}$ purchase failed predominantly by suture pullout.

Even the location of the knots has shown to change the strength of the tendon repair [30]. Placement of the knot inside repairs were significantly stronger compared to the knots outside repair after six weeks when test in vivo but ex vivo has shown that placing them outside of the repair on the tendon surface increased the strength of the repair [49]. The placement of the suture dorsally as also shown to increase the strength of the suture 2-4 fold and more recently favoured due not risking disturbance of the synovial fluid [50].

Increasing the suture calibre has shown to increase the force in static testing and fatigue strength in dynamic testing but has not shown to improve the gap resistance. Barrie et a found that the sue of 3-0 dacron sutures increased the fatigue strength compared to 4-0 sutures a 2-3- fold [51]. Taras et al., reported increases in repair strength when suture caliber was increased from 5-0 to 2-0 [52]. Furthermore Kessler repairs improved by $167 \%$ and the strength of the doublegrasping repairs improved by $391 \%$ when $2-0$ was compared to 5-0 suture [52].

The ideal suture material for flexor tendon repair needs to be easy to use, prevent gap formation but maintain its tensile 
properties until repair has achieved strength [53]. Nonabsorbable synthesis sutures included monofilament nylon, monofilament polypropylene and braided polyester monofilament nylon have should good biocompatibility are used in repair. Monofilament polypropylene is mainly used in the peripheral sutures. Higher tensile strength and stiffness was showed with coated braided polyester than monofilament nylon and polypropylene and has shown to maintain its tensile properties at body temperature [54]. Bioabsorbable sutures have not been used as they have decreased tensile strength half-life and fear of increased tissue reaction and adhesion formation.

The circumferential epitendinous sutures were used originally to make the repair more tidy but it has now showed to improve the gap resistance and the force of the repair by $10-50 \%$ [55-58]. The strongest peripheral epitendinous suture methods have been the running lock loop stitch, halsted continuous horizontal mattress suture, cross stitch technique and horizontal mattress infrafibre methods. Epitendinous sutures are often referred to peripheral sutures because they grasp not only the epitenon but also the tendon substance and so the peripheral suture has become widely used [59-62]. The strength of the peripheral suture has been found to be strengthened by increasing suture purchase from $1 \mathrm{~mm}$ to $2 \mathrm{~mm}$ or $3 \mathrm{~mm}$ an increasing the number of suture passes as well as deeper suture grasps.

It is has bee stated that the annular pulleys from A1 to A5 are vital for the biomechanical flexion as they maintain the tendons close approximation to the joint axis of rotation. It has been discussed in several reports that A2 and A4 pulleys are critical and therefore should not be disregarded so surgeons should make every decision to preserve these [63]. L-shaped incisions described by Lister allows retraction of the cruciate pulley and access though the window without damaging the annular pulley. Partial release of A2 and A4 is performed to facilitate tendon surgery and allow unrestricted tendon gliding [64]. The A2 plate can be reconstructed using free tendon graft with plantaris or palmaris longus, extensor reticulum, volar plate or fascia lata [65].

The zone in which the injury occurs can determine the surgical intervention used. Zone I injuries involve the FDP tendons only. Up to the 1960s, injuries in Zone II were not primarily repaired with the zone being referred to as "no mans land [66]. However, since then much research has been done in this area and with improved techniques and suture materials primary repair of the flexors tendon in the digital sheath has replaced the 'no mans land' consensus [66]. Zone II must be carefully treated as there is a close relationship with the FDS and the FDP, which can cause adhesion formation and failed repair. It is now recommended that both the FDS and FDP should be repaired in zone 2 injuries rather than just the flexor digitorum profundus alone as it was once thought [67]. Furthermore care should be taken by the surgeon to avoid disrupting Campers chiasm, which can be carried out by repairing only one slip of the flexor digitorium superficialis tendon and excising the other [68].

Flexor sheath and pulley management is another important debated area for the hand surgeon. To date there have been conflicting evidence whether repairing the tendon sheath is of any advantage [69]. As repair may limit tendon gliding by decreasing the overall width of the sheath.
However, the advantages are that it serves as a barrier to the formation of extrinsic adhesions, provides a quicker return of synovial nutrition and results in better tendon sheath biomechanics [70]. Zone III and V injuries have been less researched as they are less difficult to treat. The prognosis is better for these zones as better vascularity and more space in the anatomical surgical operative site. However, Zone V injuries can be complicated as isolated tendon injuries are uncommon and often nerve and artery damage is present, commonly referred to as a spaghetti wrist, which needs to be treated without any hesitant delay. Zone IV injuries are difficult as they are close the carpal tunnel which can disrupt the median nerve and arterial injury and often the transverse carpal ligament may need to be divided by stair step division to prevent bow-stringing of the tendons at the wrist. All injuries should be placed in a bulky dressing and splinted postopertively, with the wrist in 20 degrees of flexion and the metacarpal phalangeal joints flexed to 70 degrees with interphalangeal joints in extension.

Tendon devices have also been invented to improve biochemical characteristics, which compare with those of suture repair. Mersilene mesh sleeve attached with cross stitch peripheral sutures, Dacron splint and interal stainless steel anchor were not found to be suitable for clinical use despite increasing the repair strength [71-73]. Su et al., introduced the stainless Teno fix-repair device and compared 67 patients with zone 2 injury treated with the device and four-stranded cruciate suture repair [74]. There were 85 injured digits, 34 were treated with Teno fix, and fifty-one served as controls. Nine of the suture repair ruptures but none of the Teno fix $(p<0.01)$ [74]. There was no difference in range of motion, though the Teno fix group has slightly lower resolution of pain and swelling. At 6 months follow up 16 of the 24 treated with a Teno fix repair and 19 of the 27 treated with a control had a good or excellent results [74]. Despite new devices no device has been developed which has come into common clinical use to date.

Tendon grafts can be an option when a primary repaired tendon has failed after a period of exercise or when seeing a patient 3 or 4 weeks after an injury or a patient with extensive tissue loss and the surgeon feel primary repair not applicable. In the 1950s bassett and Carooll used flexible silicone rubber rods to build pseudosheath in scarred finger [75]. Which was latter developed and refined by Hunter and Salisabury into a two stage reconstruction technique which has been widely adopted today [76]. Hunter's reconstruction involves using a silicon rod in the first stage and free tendon grafter though the pseuodsheath formed around the silicone in the second stage [76].

\section{REHABILITATION}

The aim of rehabilitation after tendon repair is to achieve function and gliding but avoiding rupture of the tendon. Since the first protocol by Kleinert et al., there have been many publications presenting novel protocols but the ideal protocol, which gives the best functional outcome, is still under debate.

All rehabilitation methods vary in their protocol but there are 3 main methods. Firstly there is active extension with rubber band flexion, also called the active extension-passive flexion method [77]. Secondly there is passive motion 
method, which uses a range of $3-5 \mathrm{~mm}$ of passive motion in the involved tendon and then lastly there is controlled passive motion with the patient actively flexing the digit rather than someone else [77, 78]. There are variations of these methods in clinical practise today. The once popular rubber band technique made known by Kleinert et al., has now been favoured against by passive and active motion protocols [79]. Mobilized tendons have shown to heal quicker and are stronger with fewer adhesions than immobilised tendons [80]. Programs involve a step-wise progression of passive movements to reduce adhesion but preventing excessive loading and the active motion to overcome stiffness and swelling of digits. In one recent systematic review it was concluded that early active protocols and combined Kleinert (passive flexion and active extension) and Durran (controlled passive motion) protocols resulted in low rates of tendon ruptures and acceptable range of motion following flexor tendon repair in zone 2 [81]. Patient compliance is vitally important or success of flexor tendon repairs as patients who negatively ignore the rehabilitation protocols can impact on their results. The trend to more active mobilisation seems to be favoured but further study in this area is needed.

\section{COMPLICATIONS AFTER SURGERY}

Despite the research that has been commenced in flexor tendon repair there are still several complications that can occur with tendon surgery including adhesion formation, tendon rupture and stiffness of the joints [16]. The immediate complications can include infection, tendon rupture, pulley rupture and poor tendon gliding. Infection rates are low but this has not been studied throughout but antibiotics prophactically have shown to be beneficial [82]. Tendon rupture is the worst complication following surgery for the surgeons, as it requires urgent secondary surgical intervention, which usually can occur in 3-9\% of cases [83]. Rupture can be due to overload of the tendons, oedema, misuse of the hand or bulky tendons. Rupture is usually seen if there is a gap, as this weakens the tendon, and increases the length. Active range of motion has shown to prevent adhesion but they may be associated with an increased rate of rupture.

Later complications are also seen including tendon adhesions, decreased flexion strength, complex regional pain syndrome and abnormal scars. It has been documented the up to $20 \%$ of patients will develop adhesions which requires tenolysis or tendon grafts [84]. Formation of adhesions cannot be avoided completely and therefore tenolysis is still an excellent salvage way of releasing adhesions around the tendon repair in certain circumstances. Tenolysis is usually performed 3-6 months after initial tendon surgery and the area of release should try to give free movement [85]. Recently biological methods have been invented to decrease adhesion formation; including 5-fluorouracil and Hyaloglide, though there are still under investigation [86,87]. Stiffness of the DIP and PIP joints is common after Langlias et al., reported $19 \%$ of 68 patients treated developed stiffness after zone II lacerations [88]. Rubber band traction has decreased the rates of adhesion but surgeons still place the joints in splints so further research is required to decrease stiffness.

Bowstringing of the flexor tendons can reduce the functional outcome following flexor surgery. Loss of A2 and
A4 pulleys results in mechanical inefficiency as the flexor tendon bowstrings across the interphalangeal metacarpophalangeal joint, causing weakness. Conservative therapy for bowstring can only be achieved with pulley reconstruction and not with a pulley ring [89]. Stiffness is also common as well contractures following tendon surgery. This can be treated by rehabilitation protocols initiated early interphalangeal joint extension. Joint contracture persistently may need capsulotomy. The pathology behind complex regional pain syndrome is still unknown and its characteristics can be very variable including burning, aching, throbbing and pain.

\section{THE FUTURE OF TENDON REPAIR}

Tendon repair is still no fully understood but ways help flexor tendon repair is currently extensive. External devices have been used to stimulate tendon healing including electrical simulation by direct current, capacitive coupling and pulsed electromagnetic stimulation [90-92]. Laser therapy on tendon healing has also been studies as it increases collagen produces in rabbit after tenotomy but in flexor tendon repairs there was no improvement in grip strength or functional results of controls [93]. The use of growth factors and cytokines to enhance tendon healing still remains restricted to animal and in vitro studies [94, 95]. Animal studies have also successfully transmitted BMP-12, PDGF-B and decorin to improve healing of tendiopathy and showed the healing environment could be manipulated more in depth studies are needed [96-98]. The use of Mesenchymal stem cells (MSCs) for tendon repair has been investigated but the identification of definitive biomarkers for tendons differentiation is difficult as well few case of ectopic bone and tumour formation after transplantation of MSCs [99]. Hence tissue engineering for tendon repair is till in its inventory stage but will be in the future.

\section{CONCLUSIONS}

It is clear that there have been advancements in tendon repair with new surgical approaches being implemented over the years. However it is clear there are some disagreements on the optimal number of repair strands and the ideal suture configuration. We can expect further studies indentifying the ideal suture technique for the lacerated tendon. Furthermore rehabilitation programs have began to adopt more active approaches though more research is needed in this area.

\section{ACKNOWLEDGEMENT}

None declared.

\section{CONFLICT OF INTEREST}

None declared.

\section{REFERENCES}

[1] Kannus P, Jozsa L, Jarvinnen M. Basic science of tendons. In: Garrett WE Jr, Speer KP, Kirkendall DT, Eds. Principles and practice of orthopaedic sports medicine. Philadelphia: Lippincott Williams and Wilkins 2000; pp. 21-37.

[2] O'Brien M. Structure and metabolism of tendons. Scand J Med Sci Sports 1997; 7: 55-61.

[3] Hess GP, Cappiello WL, Poole RM, Hunter SC. Prevention and treatment of overuse tendon injuries. Sports Med 1989; 8: 371-84.

[4] Tipton CM, Matthes RD, Maynard JA, Carey RA. The influence of physical activity on ligaments and tendons. Med Sci Sports 1975; 7:165-75. 
[5] Astrom M. On the nature and etiology of chronic achilles tendinopathy. PhD dissertation. Lund, Sweden: University of Lund 1997.

[6] Jozsa LG, Kannus P. Spontaneous rupture of tendons. In: Jozsa LG, Kannus P, Eds. Human tendons: anatomy, physiology, and pathology. Champaign, IL: Human Kinetics 1997; pp. 254-325.

[7] Movin T, Kristoffersen-WM, Shalabi A, Gad A, Aspelin P, Rolf C. Intratendinous alterations as imaged by ultrasound and contrast medium-enhanced magnetic resonance in chronic achillodynia. Foot Ankle Int 1998; 19: 311-7.

[8] Kvist M, Jozsa L, Jarvinen M, Kvist H. Fine structural alterations in chronic Achilles paratenonitis in athletes. Pathol Res Pract 1985; 180: 416-23.

[9] Moutiet F. Flexor tendon pulley system: anatomy, pathology, treatment. Chir Main 2003; 22(1): 1-12.

[10] Green DP, Pederson WC, Hotchkiss RN, Wolfe SW, Eds. Greens operative Hand Surgery. $5^{\text {th }}$ ed. Philadelphia, Pennsylvania: Elsevier Churchill 2005.

[11] Kvist M, Jozsa L, Kannus P, et al. Morphologyand histochemistry of the myotendineal junction of the rat calf muscles. Histochemical, immunohistochemical and electron-microscopic study. Acta Anat (Basel) 1991; 141: 199-205.

[12] Michna H. A peculiar myofibrillar pattern in the murine muscletendon junction. Cell Tissue Res 1983; 233: 227-31.

[13] Benjamin M, Ralphs JR. Fibrocartilage in tendons and ligamentsan adaptation to compressive load. J Anat 1998; 193: 481-94.

[14] Tillman LJ, Chasan NP. Properties of dense connective tissue and wound healing. In: Hertling D, Kessler RM, Eds. Management of common musculoskeletal disorders: physical therapy principles and methods. $3^{\text {rd }}$ ed. Philadelphia: Lippincott 1996; pp. 8-21.

[15] Hooley CJ, Cohen RE. A model for the creep behaviour of tendon. Int J Biol Macromol 1979; 1: 123-32.

[16] Ting J. Tendon injuries across the world. Injury 2006; 37:1036-42

[17] Kleinert HE, Spokevicius S, Papas NH. History of flexor tendon repair. J Hand Surg Am 1995; 20: S46-52.

[18] Bruner JM. The zig-zag volar-digital incision for flexortendon surgery. Plast Reconstr Surg 1967; 40: 571.

[19] Hall RF Jr, Vliegenthart DH. A modified midlateral incision for volar approach to the digit. J Hand Surg Br 1986; 11(2):195-7.

[20] Strickland JW. Flexor tendon injuries: I. Foundations of treatment. J Am Acad Orthop Surg 1995; 3: 44-54.

[21] Barrie KA, Tomak SL, Cholewicki J, Wolfe SW. The role of multiple strands and locking sutures on gap formation of flexor tendon repairs during cyclical loading. J Hand Surg Am 2000; 25: 714-20.

[22] Hatanaka H, Manske PR. Effect of suture size on locking and grasping flexor tendon repair techniques. Clin Orthop Relat Res 2000; 375: 267-74.

[23] Hotokezaka S, Manske PR. Differences between locking loops band grasping loops: effects on 2-strand core suture. J Hand Surg Am 1997; 22: 995-1003.

[24] Komanduri M, Phillips CS, Mass DP. Tensile strength of flexor tendon repairs in a dynamic cadaver model. J Hand Surg 1996; 21 : 605-11.

[25] Manske PR, Lesker PA. Flexor tendon nutrition. Hand Clinics $1985 ; 1: 13-24$.

[26] Savage R, Risitano G. Flexor tendon repair using the six strand method of repair and early active mobilisation. J Hand Surg British 1989; 14: 369-99.

[27] Wada A, Kubota H, Hatanaka H, et al. The mechanical properties of locking and grasping suture loop configurations in four-strand core suture techniques. J Hand Surg British 2000; 25: 548-51.

[28] Taras JS. Primary flexor tendon repair. Oper Tech Orthop 1993; 3 : 270-7.

[29] Wade PJ, Wetherell RG, Amis AA. Flexor tendon repair: significant gain in strength from the halsted peripheral suture technique. J Hand Surg 1989; 14: 232-5.

[30] Savage R. In vitro studies of a new method of flexor tendon repair. J Hand Surg 1985; 10B:135-41.

[31] Barrie KA, Tomak SL, Cholewicki J, et al. Effect of suture locking and suture caliber on fatigue strength of flexor tendon repairs. J Hand Surg 2001; 26A: 340-6.

[32] Sanders DW, Milne AD, Dobravec A, et al. Cyclic testing of flexor tendon repairs: an in vitro biomechanical study. J Hand Surg 1997; 22A: 1004-10.
[33] Lee H. Double loop locking suture: a technique of tendon repair for early active mobilization. Part I: evolution of technique and experimental study. J Hand Surg 1990; 15A: 945-52.

[34] Lim BH, Tsai TM. The six-strand technique for flexor tendon repair. Atlas Hand Clin 1996; 1: 65-76.

[35] Dovan TT, Ditsios KT, Boyer MI. Eight-strand core suture technique for repair of intrasynovial flexor tendon lacerations Tech Hand Up Extrem Surg 2003; 7(2): 70-4.

[36] Viinikainen A, Göransson $H$, Huovinen $K$, Kellomäki $M$, Rokkanen P. A comparative analysis of the biomechanical behaviour of five flexor tendon core sutures. J Hand Surg 2004; 29B: 536-43.

[37] Shaieb MD, Singer DI. Tensile strengths of various suture techniques. J Hand Surg 1997; 22B: 764-7.

[38] Bainbridge LC, Robertson C, Gillies D, Elliot D. A Comparison of post-operative mobilization of flexor tendon repairs with "passive flexion-active extension" and "controlled active motion" techniques. J Hand Surg 1994; 19B: 517-21.

[39] Tang JB, Shi D, Gu YQ, Chen JC, Zhau B. Double and multiple looped suture tendon repair. J Hand Surg 1994; 19B: 699-703

[40] Kusano N, Yoshizu T, Maki Y. Experimental study of two new flexor tendon suture techniques for postoperative early active flexion exercises. J Hand Surg 1999; 24B: 152-6.

[41] Winters SC, Gelberman RH, Woo SL, Chan SS, Grewal R, Seiler JG 3rd. The effects of multiple- strand suture methods on the strength and excursion of repaired intrasynovial flexor tendons: a biomechanical study in dogs. J Hand Surg 1998; 23: 97-104.

[42] Al-Qattan MM, Al-Rakan MA, Al-Hassan TS. A biomechanical study of flexor tendon repair in zone II: comparing a combined grasping and locking core suture technique to its grasping and locking components. Injury 2011; 42(11): 1300-2.

[43] Barrie KA, Tomak SL, Cholewicki J, Wolfe SW. The role of multiple strands and locking sutures on gap formation of flexor tendon repairs during cyclical loading. J Hand Surg 2000; 25A: 714-20.

[44] Pennington DG. The locking loop tendon suture. Plast Reconstr Surg 1979; 63: 648-52

[45] Hatanaka H, Manske PR. Effect of suture size on locking and grasping flexor tendon repair techniques. Clin Orthop 2000; 375: 267-74.

[46] Hatanaka H, Manske PR. Effect of the cross-sectional area of locking loops in flexor tendon repair. J Hand Surg 1999; 24A: 75160.

[47] Cao Y, Zhu B, Xie Rg, Tang JB. Influence of core suture purchase length on strength of four-strand tendon repairs. J Hand Surg 2006; 31A: 107-12.

[48] Tang JB, Zhang Y, Cao Y, Xie RG. Core suture purchase affects strength of tendon repairs. J Hand Surg 2005; 30A: 1262-6.

[49] Pruitt DL, Aoki M, Manske PR. Effect of suture knot location on tensile strength after flexor tendon repair. J Hand Surg 1996; 21A: 969-73.

[50] Manske PR, Lesker PA. Flexor tendon nutrition. Hand Clin 1985; 1: 13-24.

[51] Barrie KA, Tomak SL, Cholewicki J, Merrell GA, Wolfe SW. Effect of suture locking and suture caliber on fatigue strength of flexor tendon repairs. J Hand Surg Am 2001; 26: 340-6.

[52] Taras JS, Raphael JS, Marczyk SC, Bauerle WB. Evaluation of suture caliber in flexor tendon repair. J Hand Surg Am 2001; 26: $1100-4$.

[53] Trail IA, Powell ES, Noble J. An evaluation of suture materials used in tendon surgery. J Hand Surg 1989; 14B: 422-7.

[54] Lawrence TM, Davis TRC. A biomechanical analysis of suture materials and their influence on a four-strand flexor tendon repair. J Hand Surg 2005; 30A: 836-41.

[55] Diao E, Hariharan JS, Soejima O, Lotz JC. Effect of peripheral suture depth on strength of tendon repairs. J Hand Surg 1996; 21: 234-9.

[56] Lotz JC, Hariharan JS, Diao E. Analytic model to predict the strength of tendon repairs. J Orthop Res 1998; 16: 399-05.

[57] Diao E, Hariharan JS, Soejima O, Lotz JC. Effect of peripheral suture depth on strength of tendon repairs. J Hand Surg 1996; 21A: 234-9.

[58] Wade PJ, Muir IF, Hutcheon LL. Primary flexor tendon repair: the mechanical limitations of the modified Kessler technique. J Hand Surg 1986; 11B: 71-6 
[59] Lin GT, An KN, Amadio PC, Cooney WP. Biomechanical studies of running suture for flexor tendons in dogs. J Hand Surg 1988; 13A: 553-8.

[60] Mashadi ZB, Amis AA. Strength of the suture in the epitenon and within the tendon fibres: development of stronger peripheral suture technique. J Hand Surg 1992; 17B: 172-5.

[61] Silfverskio"ld KL, Andersson CH. Two new methods of tendon repair: an in vitro evaluation of tensile strength and gap formation. J Hand Surg 1993; 18A: 58-65.

[62] Wade PJ, Wetherell RG, Amis AA. Flexor tendon repair: significant gain in strength from the Halsted peripheral suture technique. J Hand Surg 1989; 14B: 232-05.

[63] Lin GT, Amadic PC, An KN, et al. Functional anatomy of the human digital flexor pulley system. J Hand Surg Am 1989; 14: 949-56.

[64] Kwai B, Elliot D. "Venting" or partial lateral release of the A2 and A4 pulleys after repair of zone 2 flexor tendon injuries. J Hand Surg 1998; 23B: 649-54.

[65] Mehta V, Phillips CS. Flexor tendon pulley reconstruction. Hand Clin 2005; $21: 245$.

[66] Rust RA, Eckersley R. Twenty questions on tendon injuries in the hand. Curr Orthop 2008; 22: 17-24.

[67] Grobbelaar AO, Hudson DA. Flexor tendon injuries in children. J Hand Surg Br 1994; 19(6): 696-8.

[68] Tonkin MA. Primary flexor tendon repair: surgical techniques based on the anatomy and biology of the flexor tendon system. World J Surg1991; 15(4): 452-7.

[69] Kwai B, Eliot D. Venting or partial lateral release of the A2 and A4 pulley after repair of zone 2 flexor tendon injuries. J Hand Surg 1988; 23: 649-54.

[70] Ting J. Tendon injuries across the world. Injury 2006; 37:1036-42.

[71] Aoki M, Manske PR, Pruitt DL, Larson BJ. Tendon repair using flexor tendon splints: an experimental study. J Hand Surg 1994; 19A: 984-90.

[72] Gordon L, Tolar M, Rao KT, Ritchie RO, Rabinowitz S, Lamb RP. Flexor tendon repair using a stainless steel internal anchor. Biomechanical study on human cadaver tendons. J Hand Surg 1998; 23B: 37-40.

[73] Silfverskiöld KL, Andersson CH. Two new methods of tendon repair: an in vitro evaluation of tensile strength and gap formation. J Hand Surg 1993; 18A: 58-65.

[74] Su BW, Solomons M, Barrow A, et al. A device for zone-II flexor tendon repair. Surgical technique. J Bone Joint Surg Am 2006; 88 (Suppl 1 Pt 1): 37-49.

[75] Bassett CAL, Carroll RE. Formation of tendon sheaths by silicone rod implants. Proceedings of American Society for Surgery of the Hand. J Bone Joint Surg Am1963; (45): 884.

[76] Hunter JM, Salisbury RE. Flexor-tendon reconstruction in severely damaged hands. A two-stage procedure using a silicone-dacron reinforced gliding prosthesis prior to tendon grafting. J Bone Joint Surg Am 1971; 53(5): 829-58.

[77] Boyer MI, Strickland JW, Engles DR, Sachar K, Leversedge FJ. Flexor tendon repair and rehabilitation, state of the art in 2002 . J Bone Joint Surg Am 2002; 84(9): 1684-706.

[78] Small JO, Brennen MD, Colville J. Early active mobilisation following flexor tendon repair in zone 2. J Hand Surg Brit 1989; 14(4): 383-91.

[79] Kleinert HE, Kutz JE, Atasoy E, Stormo A. Primary repair of flexor tendons. Orthop Clin North Am 1973; 4: 865-76.

[80] Tang JB. Indications, methods, postoperative motion and outcome evaluation of primary flexor tendon repairs in zone2. J Hand Surg Eur Vol 2007; 32: 118-29.
[81] Chesney A, Chauhan A, Kattan A, Farrokhyar F, Thoma A. Systematic review of flexor tendon rehabilitation protocols in zone II of the hand. Plast Reconstr Surg 2011; 127(4): 1583-92.

[82] Cassell OC, Ion L. Are antibiotics necessary in the surgical management of upper limb lacerations? Br J Plast Surg 1997; 50: 523.

[83] Elliot D, Moiemen NS, Fleming AF, et al. The rupture rate of acute flexor tendon repairs mobilized by the controlled active motion regimen. J Hand Surg Br 1994; 19: 607.

[84] Manske PR. Flexor tendon healing. J Hand Surg Br 1988; 13: 237.

[85] Strickland JW. Results of flexor tendon surgery in zone II. Hand Clin 1985; 1(1):167-79.

[86] Zhao C, Zobitz ME, Sun YL, et al. Surface treatment with 5fluorouracil after flexor tendon repair in a canine in vivo model. J Bone Joint Surg Am 2009; 91(11): 2673-82.

[87] Riccio M, Battiston B, Pajardi G, et al. Efficiency of Hyaloglide in the prevention of the recurrence of adhesions after tenolysis of flexor tendons in zone II: a randomized, controlled, multicentre clinical trial. J Hand Surg Eur 2010; 35(2):130-8.

[88] Langlais F, Gibon Y, Canciani JP, Thomine JM. Primary suturing of zone II flexor tendons (103 digits). Results and Kleinert's limitations. Ann Chir Main 1986; 5: 301-14.

[89] Mehta V, Phillips CS. Flexor tendon pulley reconstruction. Hand Clin 2005; $21: 245$.

[90] Wang CJ, Wang FS, Yang KD, et al. Shock wave therapy induces neovascularization at the tendon-bone junction. A study in rabbits. J Orthop Res 2003; 21: 984-9.

[91] Lee EW, Maffulli N, Li CK, Chan KM. Pulsed magnetic and electromagnetic fields in experimental achilles tendonitis in the rat: a prospective randomized study. Arch Phys Med Rehabil 1997; 78: 399-404.

[92] Fujita M, Hukuda S, Doida Y. The effect of constant direct electrical current on intrinsic healing in the flexor tendon in vitro. An ultrastructural study of differing attitudes in epitenon cells and tenocytes. J Hand Surg Br 1992; 17: 94-8.

[93] Reddy GK, Stehno-Bittel L, Enwemeka CS. Laser photostimulation of collagen production in healing rabbit Achilles tendons. Lasers Surg Med 1998; 22: 281-7.

[94] Chang J, Most D, Stelnicki E, et al. Gene expression of transforming growth factor beta-1 in rabbit zone II flexor tendon wound healing: evidence for dual mechanisms of repair. Plast Reconstr Surg 1997; 100: 937-44.

[95] Banes AJ, Tsuzaki M, Hu P, et al. PDGF-BB, IGF-I and mechanical load stimulate DNA synthesis in avian tendon fibroblasts in vitro. J Biomech 1995; 28:1505-13.

[96] Wolfman NM, Celeste AJ, Cox K, et al. Preliminary characterization of the biological activities of rhBMP-12. J Bone Miner Res1995; 10: S148.

[97] Nakamura N, Shino K, Natsuume T, et al. Early biological effect of in vivo gene transfer of platelet-derived growth factor (PDGF)-B into healing patellar ligament. Gene Ther 1998; 5: 1165-70.

[98] Nakamura N, Hart DA, Boorman RS, et al. Decorin antisense gene therapy improves functional healing of early rabbit ligament scar with enhanced collagen fibrillogenesis in vivo. J Orthop Res 2000; 18: 517-23.

[99] Lui PP, Rui YF, Ni M, Chan KM. Tenogenic differentiation of stem cells for tendon repair-what is the current evidence? J Tissue Eng Regen Med 2011; 5(8): e144-63. doi: 10.1002/term.424. [Epub ahead of print]. 